\title{
Resolução de Problemas e Colaboração a Distância: modelo, artefatos e sistema
}

\author{
Diego Samir Melo-Solarte ${ }^{*}$ \\ Instituto de Computação \\ Universidade Estadual de Campinas \\ Av. Albert Einstein, 1251, Campinas, SP, Brasil \\ mdiego@ic.unicamp.br
}

\author{
M. Cecília C. Baranauskas \\ Instituto de Computação \& NIED \\ Universidade Estadual de Campinas \\ Av. Albert Einstein, 1251, Campinas, SP, Brasil \\ cecilia@ic.unicamp.br
}

Resumo Neste trabalho apresentamos o modelo Aprendizagem Colaborativa Baseada em Problemas $(A C B P)$, concebido a partir de características estruturais e funcionais dos modelos de Aprendizagem Baseada em Problemas e Aprendizagem Colaborativa Apoiada pelo Computador. $O$ modelo proposto inclui em seu processo uma etapa de Análise e Clarificação do Problema, inspirada em métodos e artefatos da Semiótica Organizacional para apoiar a geração, discussão e avaliação de idéias e soluções na interpretação e discussão do problema. O modelo proposto foi desenvolvido e integrado a um sistema gerenciador de aprendizado. Resultados preliminares de situações reais de uso sugerem sua efetividade e potencial para contextos de Educação a Distância (EaD).

Palavras-Chave: Aprendizagem Baseada em Problemas, Aprendizagem Colaborativa Apoiada pelo Computador, Semiótica Organizacional, Educação a Distância.

\begin{abstract}
In this work we present the Problem-Based Collaborative Learning (ACBP), in its Portuguese acronym) model, which is based on structural and functional characteristics coming from Problem-based Learning and Computer-Supported Collaborative Learning models. The proposed model includes in its process a stage for Analysis and Clarification of the Problem inspired in methods and artifacts from Organizational Semiotics to support the generation, discussion and evaluation of ideas and solutions in the interpretation and discussion of the problem. The proposed model is developed and integrated into a Learning Management System $(L M S)$. Preliminary results of its use in real situations suggest its effectivity and potential for e-Learning contexts.
\end{abstract}

Keywords: Problem Based Learning, Computer Supported Collaborative Learning,Organizational Semiotics, E-Learning.

\footnotetext{
* Pesquisa apoiada pela Universidade de Manizales - Colômbia através de comissão de estudos e pela Fundação de Desenvolvimento da Unicamp - FUNCAMP através de bolsa de mestrado.
} 


\section{Introdução}

A presença de Tecnologias de Informação e Comunicação (TIC) na sociedade contemporânea tem gerado mudanças em muitos de seus processos; em especial processos educativos têm sido potencialmente favorecidos com a presença da Internet. Cada vez mais, as TIC são consideradas no processo de ensino e aprendizagem, para romper barreiras de tempo e distância, além de promover um processo onde as ferramentas usadas permitem uma dinâmica mais flexível e motivadora a seus atores.

Ao mesmo tempo, a inclusão dos computadores e da Internet em processos educacionais têm mexido com os modelos tradicionais da educação fornecendo grandes possibilidades para mudar sua dinâmica com ferramentas para melhor apoiar o processo de ensino e aprendizagem.

Um grande número de estudiosos da educação mediada por computadores tem defendido o conceito de atividades autênticas e contextualizadas como forma de engendrar um aprendizado significativo [25]. Popularizado nos anos 60, PBL (do inglês Problem Based Learning) tenta engajar pequenos grupos de estudantes em problemas "do mundo real" enquanto desenvolvem habilidades meta-cognitivas e de construção de conhecimento no domínio do problema. Tradicionalmente, atividades de PBL acontecem em cenários face-a-face. Em anos recentes a tecnologia da computação tem assumido o papel de suporte na mediação de atividades em PBL.

O suporte da mídia computacional a PBL tem servido a duas funções: prover o estudante com fácil acesso a recursos necessários para resolução do problema e facilitar a comunicação do grupo [10]. Enquanto essas novas funcionalidades favorecem a resolução do problema, elas não exploram todo o potencial da mídia para o processo de aprendizagem via PBL. Ferramentas de comunicação usuais (e-mail, chat, fóruns, etc.) em geral não são efetivas ou eficientes no novo contexto, uma vez que foram primariamente desenvolvidas para outros fins (discussão casual, por ex.). Vários estudos têm mostrado que essas ferramentas não são suficientes para rastrear os processos de discussão e raciocínio, determinar como os estudantes geram idéias, desenvolvem ações e planos para resolver o problema, por exemplo. Hung e Lockard [10] apontam a falta de ferramentas para guiar os estudantes enquanto eles estão engajados em processos de brainstorming, geração e trocas de idéias, compartilhamento na equipe e entre equipes.

Quando o problema em que os estudantes estão trabalhando é complexo, como acontece em problemas reais, eles precisam uns dos outros em ambientes colaborativos.
O compartilhamento de experiências, insights e entendimentos nesses ambientes pode ajudar os aprendizes na geração, discussão e avaliação de idéias e de soluções, além de tornar explícita a complexidade dos conceitos e habilidades requeridos [14]. Entendemos por ambientes colaborativos ou CSCL (do inglês Computer-Supported Collaborative Learning) formas de aprendizado contextualizadas socialmente e mediadas por Tecnologias de Informação e Comunicação. Neste trabalho a abordagem ao aprendizado baseada em problemas e mediada por ambientes computacionais colaborativos é sintetizada em um modelo conceitual que denominamos ACBP, acrônimo de Aprendizagem Colaborativa Baseada em Problemas.

O modelo ACBP resulta da avaliação, seleção e integração de características dos modelos PBL e CSCL. ACBP propõe e apóia a resolução de problemas reais por grupos de estudantes por meio da interação na Web [18].

ACBP é constituído de cinco fases entre as quais propõe artefatos específicos para a clarificação do problema e geração e discussão de idéias utilizando a Semiótica Organizacional (SO) [15]. Essa abordagem ao tratamento do problema possibilita uma perspectiva social e um contexto mais abrangente para a interpretação do problema, interação do grupo e proposição e socialização das soluções propostas.

Neste artigo apresentamos o modelo conceitual ACBP e o design e desenvolvimento do ambiente ACBPSakai. O texto está organizado da seguinte forma: a seção 2 detalha o referencial teórico que sustenta o modelo conceitual proposto; a seção 3 apresenta o modelo, sua estrutura, fases e atividades; a seção 4 analisa requisitos para instanciar o modelo em um sistema de suporte ao aprendizado e colaboração a distância; a seção 5 apresenta a arquitetura ACBP-Sakai, o conjunto de ferramentas que implementam o modelo ACBP; a seção 6 apresenta resultados preliminares de uso e a seção 7 conclui.

\section{Referencial Teórico de Base}

A literatura tem mostrado que em colaboração os aprendizes podem resolver problemas muito complexos e chegar a soluções muito mais sofisticadas do que se o fizessem sozinhos [14]. O modelo que propomos se apóia na idéia de que o compartilhamento de significados e perspectivas sobre um problema é fundamental para a geração de idéias, sua discussão e proposição de soluções, bem como para ações de meta-reflexão sobre o problema e o conhecimento construído ao lidar com ele. Nesta seção sumarizamos conceitos de PBL e CSCL e apresentamos de forma breve os artefatos da Semiótica 
Organizacional, que nos inspiraram na proposta de ferramentas para suporte à articulação compartilhada do problema.

O modelo PBL, desde seu início na escola de medicina da Universidade de Macmaster nos anos 60, foi considerado um bom esquema construtivista no processo de ensino e aprendizagem, orientado a solução de casos clínicos. Com o tempo PBL tem evoluído e tem sido adaptado a diferentes áreas de conhecimento, levando a diferentes variações com relação à sua proposta original.

Segundo Barrows [2], PBL é um método de aprendizagem baseado no principio de usar problemas como ponto de partida para a aquisição e integração de novos conhecimentos. PBL é um processo de aprendizagem centrado no aluno, $[4,11,18]$, onde atividades são organizadas em grupos pequenos de estudantes e os professores são facilitadores ou guias do processo, [4, 9].

Gómez [9] define PBL como: "um método didático, que faz parte das pedagogias ativas e particularmente das estratégias de ensino denominadas aprendizagem por descoberta e construção". Em PBL o estudante é quem se apropria do processo, procura a informação, a seleciona, a organiza e usa para tentar resolver os problemas propostos pelo professor. Este, por sua vez, é um orientador e um expositor dos problemas ou situações problemáticas, sugerindo fontes de informação e estando pronto a colaborar com as necessidades do aprendiz, [4, 18, 25].

PBL é um processo de aprendizagem centrado no estudante no qual episódios de aprendizagem são motivados por um problema inicial com alguma semelhança com problemas "do mundo real". Os problemas são mal estruturados ou de estrutura aberta $[11,25]$, para gerar e provocar conflitos cognitivos entre os estudantes [18], sendo indispensável para aprendizagens significativas [2]. O uso de PBL procura promover nos estudantes habilidades para resolver problemas a partir da interação do grupo [4, 18, 20].

A principal característica encontrada na revisão de literatura em PBL, está na ausência de um modelo com estrutura única. Os modelos PBL encontrados diferem no número de atividades do processo e na forma como as atividades são agrupadas em fases. Assim, foram encontradas propostas de modelos PBL de quatro fases [12], cinco fases [1], sete fases [5, 9], oito fases [9, 18] e nove fases [9, 23].

$\mathrm{O}$ aprendizado a partir da resolução de problema em grupo certamente requer trabalho colaborativo. CSCL é considerada uma área das ciências da aprendizagem que estuda como as pessoas podem aprender de forma conjunta e com ajuda dos computadores [6, 13, 21]. CSCL está orientado a ambientes computacionais motivadores, que permitem a aprendizagem e a construção de significados a partir das interações dos diferentes membros de um grupo, discussões, resolução de problemas, tarefas e todas aquelas situações que promovem a construção de conhecimento $[8,21]$.

A partir de levantamento da literatura em CSCL é possível identificar algumas características sugeridas para trabalho em ambientes colaborativos mediados por TIC, que envolvem:

(a) Meios apropriados de diálogo e comunicação como chat, videoconferência, teleconferência, publicação de notícias, e-mail, fóruns e editores colaborativos entre outros $[3,7,8]$.

(b) Sistemas de apoio às decisões como os fóruns com uma estrutura para a construção de conhecimento, ou seja, com um esquema que regule, armazene e articule cada participação $[3,8]$.

(c) Mecanismos adequados para gerenciamento e supervisão do processo [3]. Funções para coordenação dos tempos e atividades [8], além de permitir a assistência dos professores [7].

(d) Áreas de socialização com a disponibilidade dos recursos necessários para o diálogo [6] e diferentes tipos de indicadores, que permitam apresentar a interação e participação dos estudantes por meio de visualizações como gráficos de barras, gráficos circulares, etc. Que são utilizados nas atividades de resolução de problemas [7].

\subsection{Método e Artefatos para Articulação de Problemas}

No modelo que propomos neste trabalho, a articulação compartilhada de problemas é transversal a todo o processo, e é inspirada em métodos e artefatos da Semiótica Organizacional $[15,22]$ adaptados para uso em contexto colaborativo a distância. A SO é considerada uma disciplina da Semiótica particularmente relacionada com o tratamento da informação em seus vários níveis sígnicos, nos grupos sociais.

Neste trabalho são usados alguns dos métodos MEASUR (Methods for Eliciting, Analysing and Specifying Users Requirements) [22]: um conjunto de métodos para tratar um problema desde seus estágios iniciais de entendimento, quando suas fronteiras ainda não são claras e a solução deve considerar os aspectos sociais, pragmáticos e semânticos que o envolvem, além da infra-estrutura necessária (aspectos físicos, empíricos e sintáticos). Trabalhamos especificamente com artefatos que possibilitam a articulação do problema em seus estágios iniciais, para apoiar o compartilhamento de significados entre os participantes e informar sobre problemas das 
partes interessadas e soluções vislumbradas, que terão potencial impacto na solução. Os artefatos utilizados são:

Análise de Partes Interessadas: ajuda o grupo de participantes a entender a situação real do problema e os requisitos de solução pretendida, por meio da discussão e levantamento das partes que direta ou indiretamente influenciam ou sofrem a influência da solução.

Quadro de Avaliação: permite ao grupo identificar, para cada categoria de partes interessadas, seus interesses, principais questões e problemas, para discutir possíveis soluções que impactarão em requisitos para soluções.

Escada Semiótica: Da perspectiva semiótica várias camadas de significado devem ser consideradas na discussão de um problema. O Framework Semiótico de Stamper [22] - ou Escada Semiótica - é composto de seis camadas (ou degraus), a saber: Mundo Social, Pragmática, Semântica, Sintática, Mundo Empírico e Físico.

\section{O Modelo ACBP}

ACBP é um modelo que articula características de operação e desenvolvimento propostas pelos modelos PBL e CSCL, utilizando a SO [15] como uma abordagem transversal à análise, discussão e clarificação de problemas.

O modelo propõe uma dinâmica de trabalho colaborativo baseada na construção de conhecimento a partir das procuras de informação, aportes individuais de idéias e conceitos, além da construção de significados a partir das discussões de todos os membros do grupo, mediadas por artefatos da SO.

Assim como nos modelos PBL e CSCL, no ACBP o estudante tem papel ativo na determinação das atividades a desenvolver para solução do problema. O professor durante o processo tem o papel de facilitador, orientando os estudantes, mediando discussões e supervisionando as diferentes atividades do grupo.

\subsection{Estrutura do Modelo ACBP}

O modelo ACBP é composto por um conjunto de atividades distribuídas em cinco fases (ver Figura 1), não necessariamente sequenciais. Na Figura 1, elipses representam os repositórios como lugares de armazenamento da informação dependendo do nível de acesso. As diferentes atividades do processo estão representadas por retângulos, as setas grossas representam a relação direta entre as atividades, e as setas finas representam relações mediadas pelos repositórios.

A aplicação do modelo para resolução de um problema começa com a proposição de um problema, o planejamento das atividades por parte do professor/facilitador e a constituição dos grupos de trabalho (Fase 1).

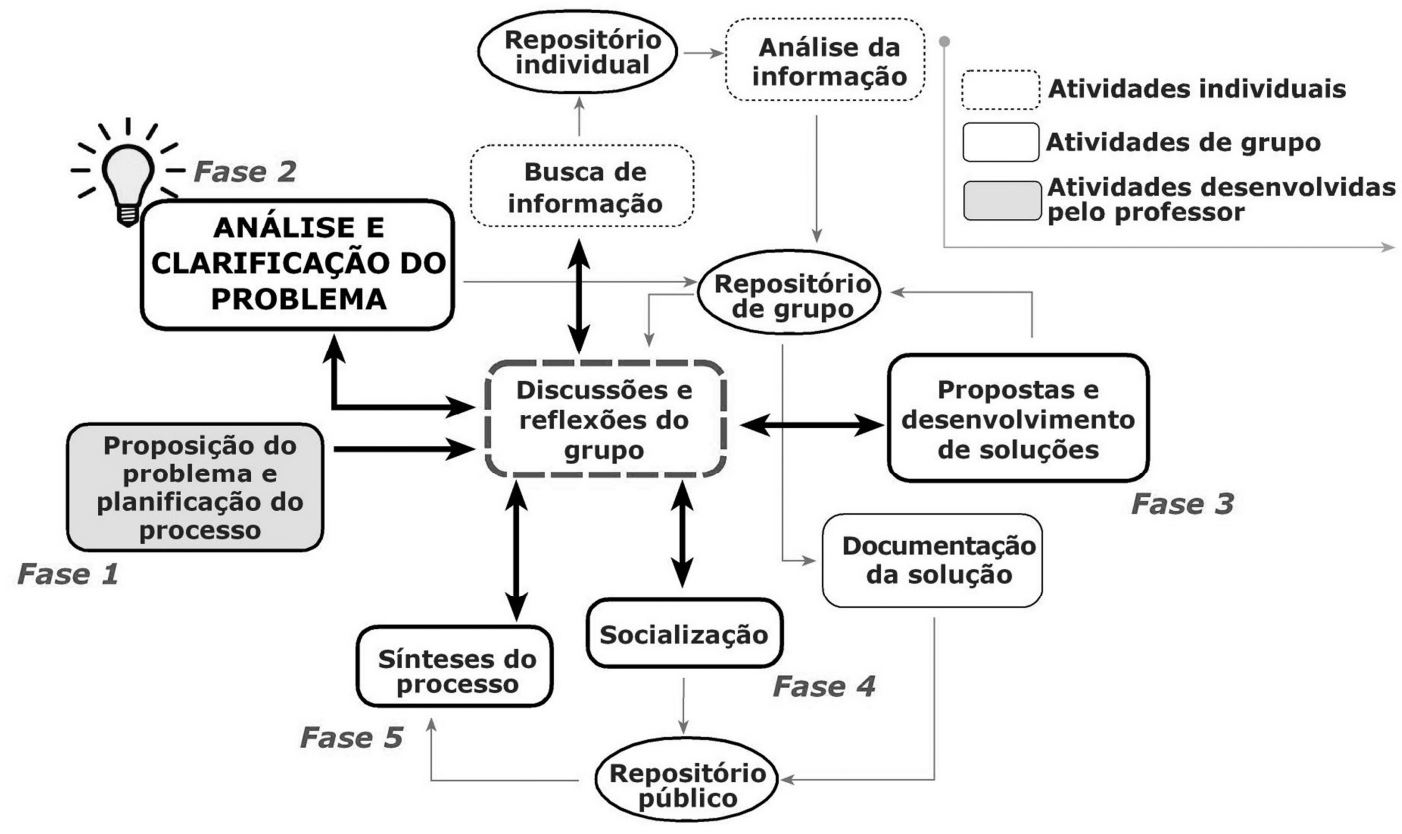

Figura 1: Diagrama do Modelo ACBP 
O desenvolvimento das soluções acontece em torno de uma atividade central chamada "Discussões e Reflexões do Grupo". As primeiras atividades que o grupo deve realizar estão determinadas pela Fase 2. Nesta fase o grupo realiza a análise e clarificação do problema utilizando artefatos que fazem parte do Método de Articulação de Problemas (PAM do inglês Problem Articulation Method) [22].

Os diferentes artefatos e documentos gerados nesta fase são armazenados no repositório de grupo como informações disponíveis para todos os membros do grupo. Cada membro pode desenvolver uma atividade de busca de informação complementar e armazená-la no repositório individual enquanto é processada e avaliada para ser compartilhada com os outros membros no repositório de grupo.

Na Fase 3, as discussões são orientadas ao trabalho das diferentes propostas e desenvolvimento de soluções armazenando os documentos criados no repositório do grupo e iterando até consolidar uma solução na qual todos os membros estão de acordo. Os documentos gerados nesta fase, antes de serem liberados, devem passar por uma atividade de documentação da solução que permite sua formalização antes de chegar ao repositório público.

Quando os documentos da solução estão no repositório público são acessíveis pelos integrantes de outros grupos. Neste momento o grupo que desenvolveu a solução pode compartilhar e socializar com outros membros do curso ou turma (Fase 4). A atividade de síntese (Fase 5) permite ao facilitador e aos diferentes membros de um grupo conhecer a evolução do processo e ter um histórico das diferentes atividades desenvolvidas possibilitando a criação de um relatório final do processo.

Tabela 1: Fases e Atividades do Modelo ACBP

\begin{tabular}{|c|c|c|}
\hline Fase & Descrição & Atividades \\
\hline $\begin{array}{l}\text { Fase } 1 \\
\text { Proposição do } \\
\text { problema e pla- } \\
\text { nificação do } \\
\text { processo }\end{array}$ & $\begin{array}{l}\text { Esta fase inicia com a proposição de um pro- } \\
\text { blema de estrutura aberta seguida de outras } \\
\text { atividades relativas ao planejamento do pro- } \\
\text { cesso. }\end{array}$ & $\begin{array}{l}\text { - } \quad \text { Proposição do problema. } \\
\text { Disponibilização de material de } \\
\text { base. } \\
\text { - } \quad \text { Elaboração de cronograma. } \\
\text { - } \quad \begin{array}{l}\text { Propoşãa de grupos de mecanismos de socia- } \\
\text { lização }\end{array}\end{array}$ \\
\hline $\begin{array}{l}\text { Análise e Clari- } \\
\text { ficação do Pro- } \\
\text { blema }\end{array}$ & $\begin{array}{l}\text { A análise e clarificação do problema são } \\
\text { feitos empregando o Método de Articulação de } \\
\text { Problemas que reúne um conjunto de procedi- } \\
\text { mentos a serem aplicados no estágio inicial } \\
\text { de um problema, quando ele é ainda vago e } \\
\text { complexo. Esse método provê artefatos que } \\
\text { permitem aos estudantes encontrar e detalhar } \\
\text { caracteristicas que requeiram atenção duran- } \\
\text { te o desenvolvimento do problema. }\end{array}$ & $\begin{array}{l}\text { - Diálogo inicial de aproximação e } \\
\text { - } \quad \text { Coordenação das atividades. } \\
\text { postos ou requisitos do problema. } \\
\text { - Criação do cronograma do grupo, } \\
\text { atividades de busca de informa- } \\
\text { Ção. } \\
\text { - Articulação do problema. }\end{array}$ \\
\hline $\begin{array}{l}\text { Propostas e De- } \\
\text { senvolvimento de } \\
\text { Soluções }\end{array}$ & $\begin{array}{l}\text { Os grupos dialogam sobre os achados indivi- } \\
\text { duais e constroem as soluções do grupo para } \\
\text { o problema. } \\
\text { Nesta fase não se pretende obter uma solução } \\
\text { concreta e definitiva, no entanto pretende- } \\
\text { se detalhar e argumentar sobre possíveis } \\
\text { soluções. } \\
\text { Cada um dos integrantes deve defender sua } \\
\text { proposta; isto permite que o estudante tra- } \\
\text { balhe habilidades de comunicação e expressão } \\
\text { e assim assuma posições críticas frente ao } \\
\text { problema e à sua proposta. }\end{array}$ & $\begin{array}{l}\text { - } \quad \text { Diálogo sobre os documentos com- } \\
\text { - } \quad \text { proptilhados e informe individual. } \\
\text { discussões de idéias de solução, } \\
\text { problema. } \\
\text { - } \quad \text { anstrução da solução. } \\
\text { - Aprovação da solução. }\end{array}$ \\
\hline Socialização & $\begin{array}{l}\text { A Fase de Socialização envolve atividades } \\
\text { nas quais cada grupo expõe à crítica dos } \\
\text { demais atores (professor e outros estudan- } \\
\text { tes) sua solução para o problema. }\end{array}$ & $\begin{array}{l}\text { - Apresentação da solução por parte } \\
\text { de cada grupo. } \\
\text { Comentários por parte da turma e } \\
\text { do professor. } \\
\text { - Respostas e argumentos de grupo. } \\
\text { - Recomendações da turma e do pro- } \\
\text { fessor. }\end{array}$ \\
\hline $\begin{array}{l}\quad \text { Fase } 5 \\
\text { Sintese do pro- } \\
\text { cesso }\end{array}$ & $\begin{array}{l}\text { Esta fase provê atividades para consolidação } \\
\text { e síntese de resultados obtidos pelo grupo } \\
\text { no processo ACBP orientadas à divulgação de } \\
\text { seu produto. }\end{array}$ & $\begin{array}{l}\text { - } \quad \text { Consolidação do documento da } \\
\text { solução com as recomendações. } \\
\text { - Síntese da lista de atividades. } \\
\text { - Publicação. }\end{array}$ \\
\hline
\end{tabular}


Na Tabela 1 são apresentadas as fases do modelo conceitual ACBP com as atividades propostas para cada uma delas.

\section{Instanciação do Modelo ACBP}

A seguir apresentamos um conjunto de parâmetros sobre os quais foram baseadas as avaliações de alguns sistemas LMS (do inglês Learning Management Systems), que permitiram identificar os que melhor poderiam apoiar a implementação do modelo ACBP. Em seguida apresentamos a forma como o modelo ACBP foi instanciando no ambiente escolhido e que novas ferramentas foram desenvolvidas e incorporadas ao ambiente.

\subsection{Análise de ambientes de suporte a EaD}

Atualmente existem vários ambientes de suporte a $\mathrm{EaD}$, os LMS; neste trabalho procuramos ambientes que estivessem dentro do conjunto das licenças GNU (General Public License) e que permitissem a reutilização de seu código fonte para implementar novas ferramentas. Os ambientes escolhidos para avaliação foram: Atutor ${ }^{1}$, Fle $^{2}{ }^{2}$, Moodle ${ }^{3}$, Sakai $^{4}$ e Teleduc ${ }^{5}$. O objetivo era determinar em que medida eles poderiam instanciar o modelo ACBP. A seguir apresentamos um conjunto de parâmetros sobre os quais foram baseadas as avaliações, que permitiram identificar as principais características de cada ambiente e apoiar a escolha.

$\mathrm{Na}$ primeira etapa da avaliação, foram identificadas algumas características técnicas de cada um dos ambientes (ver Tabela 2).

Tabela 2: Características técnicas de LMS analisados

\begin{tabular}{|l|c|c|c|c|}
\hline & Versão & Linguagem & $\begin{array}{c}\text { Servidor } \\
\text { WEB }\end{array}$ & DBMS \\
\hline Atutor & 1.6 & PHP & Apache & MySql \\
\hline Fle3 & 3.0 & Python & Zope & Gadfly \\
\hline Moodle & 1.9 & PHP & Apache & MySql \\
\hline Sakai & 2.4 & JAVA/JSP & Tomcat & MySql \\
\cline { 2 - 5 } Teleduc & 4.0 & PHP & Apache & MySql \\
\hline
\end{tabular}

\footnotetext{
${ }^{1}$ http://www.atutor.ca

${ }^{2}$ http://fle3.uiah.fi

${ }^{3} \mathrm{http}: / /$ moodle.org

${ }^{4} \mathrm{http} / / /$ sakaiproject.org

${ }^{5}$ http://www.teleduc.org.br
}

Na segunda etapa da avaliação, identificamos para o conjunto de ferramentas requeridas pelo modelo ACBP, o ambiente que estivesse mais próximo do proposto no modelo.

As ferramentas requeridas pelo modelo ACBP foram listadas e classificadas por fase. Inicialmente, avaliou-se nos ambientes a existência ou não das ferramentas (ver Tabela 3), independentemente da flexibilidade ou possibilidades de configuração exigidas pelo modelo.

Baseados no resultado desta análise observamos que Fle3, embora interessante para trabalho colaborativo, apresenta deficiências quanto à disponibilidade de ferramentas requeridas pelo ACBP. Atutor, Moodle, Sakai e Teleduc fornecem um bom número de ferramentas para a instanciação do modelo ACBP.

Tais ambientes, embora apresentem um bom número de ferramentas para o processo de $\mathrm{EaD}$ e para apoiar a aprendizagem colaborativa, não apresentam recursos para apoiar atividades como a análise e clarificação de problemas na forma como proposta no ACBP (Fase 2 do modelo ACBP) e síntese do processo (Fase 5 do modelo ACBP) que permitam construir um relatório final.

Tabela 3: Ferramentas requeridas pelo modelo ACBP

\begin{tabular}{|c|c|c|c|c|c|}
\hline $\begin{array}{l}\text { Ferramenta } \\
\text { requerida }\end{array}$ & Atutor & Fle3 & Moodle & Sakai & Teleduc \\
\hline $\begin{array}{l}\text { Gestor de } \\
\text { conteúdos }\end{array}$ & $\checkmark$ & $\checkmark$ & $\checkmark$ & $\checkmark$ & $\checkmark$ \\
\hline Glossário & $\checkmark$ & $\checkmark$ & $\checkmark$ & $\checkmark$ & $\checkmark$ \\
\hline Agenda & $\checkmark$ & & $\checkmark$ & $\checkmark$ & $\checkmark$ \\
\hline $\begin{array}{l}\text { Ferramentas } \\
\text { para apoiar o } \\
\text { PAM }\end{array}$ & & & & & \\
\hline Fórum & $\checkmark$ & $\checkmark$ & $\checkmark$ & $\checkmark$ & $\checkmark$ \\
\hline Enquetes & $\checkmark$ & & $\checkmark$ & $\checkmark$ & $\checkmark$ \\
\hline Chat & $\checkmark$ & & $\checkmark$ & $\checkmark$ & $\checkmark$ \\
\hline Wiki & & & $\checkmark$ & $\checkmark$ & $\checkmark$ \\
\hline $\begin{array}{l}\text { Gerador de } \\
\text { relatório }\end{array}$ & & & & & \\
\hline Repositório & $\checkmark$ & $\checkmark$ & $\checkmark$ & $\checkmark$ & $\checkmark$ \\
\hline
\end{tabular}

Na terceira fase da avaliação, verificamos a flexibilidade de configuração disponível para as ferramentas 
fornecidas pelos ambientes Atutor, Moodle, Sakai e Teleduc (ver Tabela 4). A flexibilidade de configuração é necessária em termos de possibilidades para usar as ferramentas em qualquer nível de acesso (individual, grupal ou de turma), pois o modelo conceitual ACBP requer estes tipos de espaços para seu desenvolvimento.

No nível individual, identifica-se o estudante como um ator independente que requer um espaço para administrar os diferentes recursos ou elementos por ele gerados. No nível de grupo, pretende-se dividir a turma em conjuntos de três ou quatro estudantes para desenvolver um problema e disponibilizar um espaço de trabalho no qual possam interagir com todos os membros do grupo. No nível de turma, pretende-se disponibilizar um espaço de trabalho onde todos os integrantes do curso (ou disciplina), possam interagir e compartilhar os documentos com as propostas de solução.

Para este requisito do modelo, foi possível identificar que os quatro ambientes disponibilizam um módulo para administrar e gerenciar grupos, mas nem todos eles fornecem a possibilidade de configurar cada ferramenta para criar um espaço de trabalho em grupo.

Na Tabela 4, exibimos a possibilidade de personalização das ferramentas requeridas pelo modelo ACBP frente ao que possibilita cada ambiente, apresentando a possibilidade para criar espaços de trabalho individual (I), em grupo (G) e na turma (T). Posteriormente quantificamos os resultados, assinalando um ponto ao ambiente para cada espaço de trabalho possibilitado.

Tabela 4: Flexibilidade de cada ferramenta para possibilitar espaços de trabalho individual, em grupo e turma.

\begin{tabular}{|l|l|l|l|l|}
\hline & Atutor & Moodle & Sakai & Teleduc \\
\hline $\begin{array}{l}\text { Gestor de } \\
\text { conteúdos }\end{array}$ & T & T & I, G, T & T \\
\hline Glossário & T & T & I, G, T & T \\
\hline Agenda & T & I, G, T & I, G, T & T \\
\hline Chat & T & G, T & T & - \\
\hline Fórum & I, G, T & T & I, G, T & T \\
\hline Repositório & I, G, T & T & I, G, T & I, G, T \\
\hline Wiki & - & I, G, T & T & - \\
\hline Enquetes & T & T & T & T \\
\hline Pontuação & $\mathbf{1 1}$ & $\mathbf{1 3}$ & $\mathbf{1 8}$ & $\mathbf{8}$ \\
\hline
\end{tabular}

Feita esta análise, foi possível identificar o Sakai como o ambiente que está mais próximo das características funcionais e operacionais necessárias à instanciação do modelo ACBP. O Sakai apresenta um evidente esquema de trabalho em grupo aplicado em suas ferramentas, que é um aspecto fundamental no ACBP.

A última fase da avaliação foi feita sobre o Sakai e o Moodle, que são os ambientes que evidenciam a maior tendência ao trabalho em grupo. Buscamos identificar a possibilidade fornecida por eles para implementar e incluir novos módulos, permitindo ativá-los ou desativálos a qualquer momento. O Sakai conta com a ferramenta Link Tools, que permite inserir sites ou páginas web externas, como módulos que fazem parte do ambiente. Por padrão, Sakai permite inserir um site externo no ambiente, mas é possível editar o código fonte da ferramenta Link Tools e permitir a inclusão de vários sites, cada um independente, com a possibilidade de ser habilitado ou desabilitado, dependendo das necessidades do administrador ou professor.

O Moodle permite implementação de novos elementos, mas a diferença com relação ao Sakai está em que as funcionalidades devem ser empacotadas como um plugin a ser instalado dentro do ambiente. Conta com diferentes API's fornecidas pelo grupo desenvolvedor, que já tem implementado muitas funcionalidades básicas e que ajudam no desenvolvimento do novo elemento.

Outros aspectos importantes levados em consideração na escolha do ambiente foram o suporte fornecido pelos ambientes, a popularidade, a projeção e a facilidade de interação. Finalmente, foi escolhido o Sakai como o ambiente a ser usado para instanciar o modelo ACBP, utilizando-se a ferramenta Link Tools, para integração de quatro módulos adicionais ao Sakai e que são requeridos pelo modelo ACBP: Modelo, PAM, Documentos e Reuniões, descritos na próxima seção..

\subsection{Arquitetura do ACBP - Sakai}

Sakai e o modelo ACBP articulam-se em uma estrutura de componentes, onde cada uma das ferramentas requeridas pelo modelo é denominada módulo e é fornecida pelo ambiente ou desenvolvida como ferramenta complementar ao modelo, usando Link Tools (ver Figura 2).

Link Tools é um módulo fornecido pelo Sakai que permite reconhecer as ferramentas do ACBP e inseri-las no Sakai. Por sua vez, ACBP usa três componentes (User, Site e Authz) para poder identificar os usuários cadastrados, os papéis, as permissões e os grupos, elementos necessários para identificar e criar uma sessão.

Para manter a independência de plataforma do ACBP, foram implementados três componentes, dois dos quais (ACBPUser e Socialize) são usados para gestão das 
sessões de cada usuário, e um terceiro componente (Connection) é usado para permitir acesso ao banco de dados, que pode ser implementado em PostgreSQL ou Mysql, mudando apenas a biblioteca de conexão de Java Database Connectivity (JDBC).

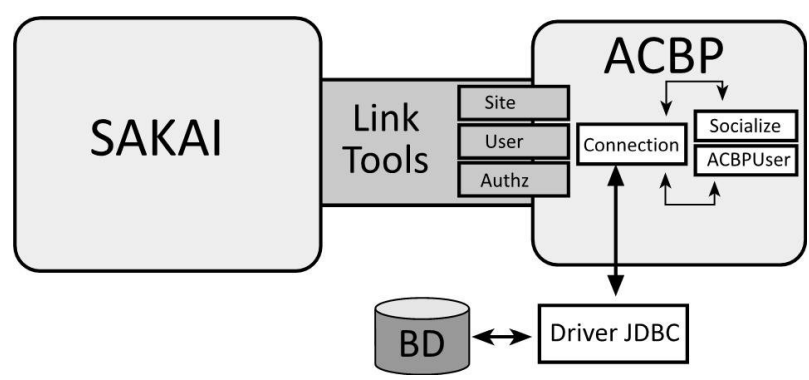

Figura 2: Arquitetura do modelo ACBP instanciado no Sakai

As ferramentas de ACBP, descritas na próxima seção, foram implementas usando Java/JSP como linguagem de desenvolvimento cliente/servidor. Para o desenvolvimento das interfaces de usuário, foi usado Javascript com a biblioteca ExtJS ${ }^{6}$ e Cascading Style Sheets $(\mathrm{CSS})^{7}$.

\section{O ambiente ACBP-Sakai}

Em síntese, o modelo ACBP integrado ao Sakai, (denominado ACBP-Sakai) é composto de um conjunto de 14 ferramentas base (ver Figura 3), que permitirão desenvolver e apoiar ACBP a distância.

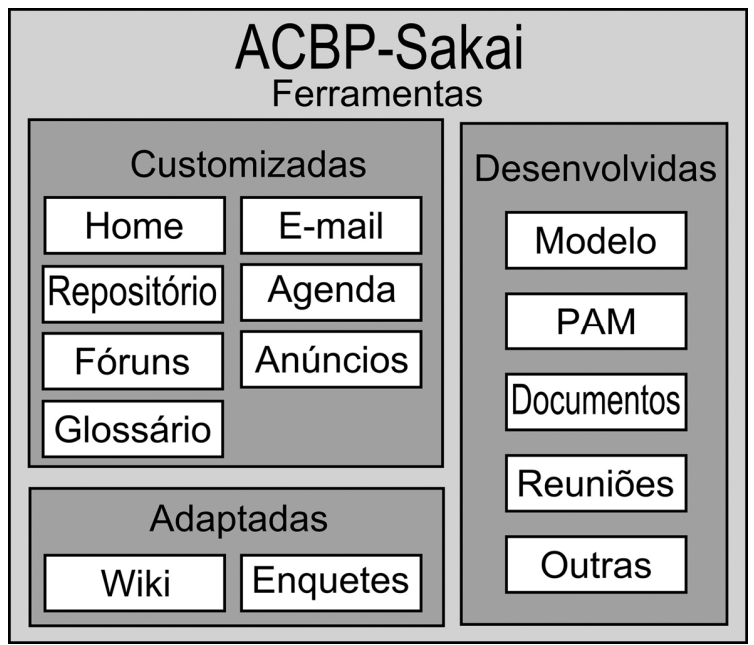

Figura 3: Ferramentas disponíveis no ACBP-Sakai
A ferramenta Home permite disponibilizar conteúdos informativos na primeira tela do ambiente.

As ferramentas Anúncios, Agenda, Repositório, Fóruns, E-mail e Glossário foram customizadas do Sakai para permitir espaços de trabalho individuais, grupais e de turma.

A ferramenta Wiki, usada para construção de documentos e a ferramenta Enquetes, usada para votações, permitiam a interação de todos os membros da turma, mas não permitiam o trabalho em grupo. Para tal foi necessário editar o código fonte existente, para desenvolver novas funcionalidades e cumprir com os requisitos do modelo ACBP. Sakai conta com outras ferramentas para apoiar diferentes atividades colaborativas, mas não era objetivo deste trabalho gerar um compêndio de ferramentas, senão identificar aquelas que poderiam instanciar o modelo ACBP, sem sobrecarregar ou saturar o conjunto de funcionalidades requeridas pelos usuários do ACBP.

$\mathrm{Na}$ arquitetura ACBP-Sakai, identificou-se a necessidade de quatro novos módulos para satisfazer os requisitos do modelo ACBP, são eles: Modelo, PAM, Reuniões e Documentos.

Cada módulo é composto por novas ferramentas que são disponibilizadas por meio de uma interface de usuário baseada na estrutura de abas (ver Figura 4). Basicamente, cada módulo disponibiliza uma interface de usuário composta por três regiões. Uma região central principal, onde é disponibilizada cada uma das ferramentas, podendo-se mudar entre ferramentas por meio da navegação por abas; uma região auxiliar na parte direita, que permite disponibilizar a área de informação e a área de chat; uma região auxiliar na parte inferior, que disponibiliza a área para construção compartilhada da interpretação do problema. Os espaços auxiliares podem ser ocultados segundo a necessidade ou interesse do usuário.

A seguir são apresentados os módulos e cada uma das novas ferramentas desenvolvidas para a instanciação do modelo ACBP no Sakai.

\footnotetext{
${ }^{6}$ http://extjs.com

${ }^{7}$ http://www.w3schools.com
} 


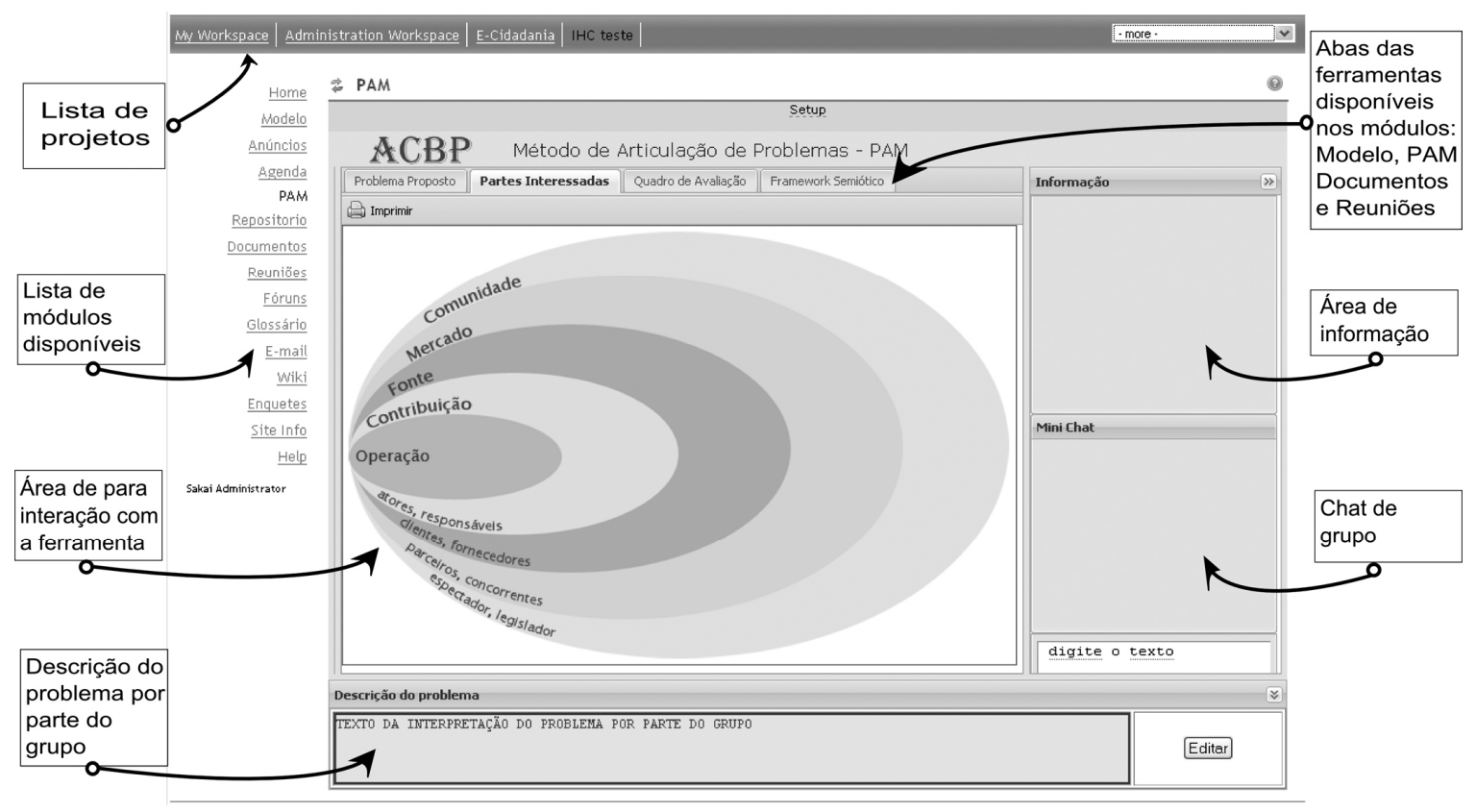

Figura 4: Interface de usuário do ACBP-Sakai com artefato de Partes Interessadas em destaque

\subsection{Módulo Modelo}

Este módulo conta com cinco componentes informativos, que objetivam apresentar as características do modelo e exemplificar sua aplicação (ver Figura 5). Compõem esse módulo as seguintes abas:

Descrição: nesta aba estão postadas informações que definem o modelo conceitual ACBP e sua natureza.

Arquitetura: esta aba permite conhecer a estrutura do modelo ACBP identificando cada uma de suas fases e as relações existentes entre elas.
Atividades: nesta aba estão descritas as características e as atividades que fazem parte de cada uma das fases do ACBP.

Sugestões: mostra informações relacionadas a como pode ser aplicado o modelo ACBP em uma disciplina.

Exemplo: apresentada um estudo de caso para exemplificar a forma como o modelo conceitual é aplicado e a forma de interagir com as ferramentas e o ambiente em geral.

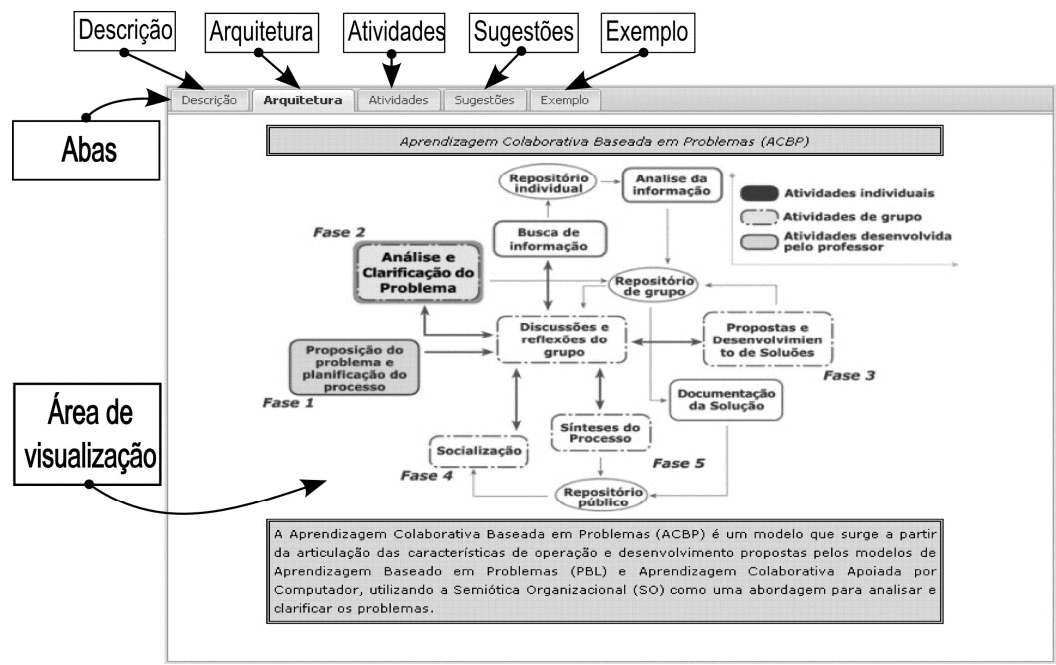

Figura 5: Interface de usuário - aba Arquitetura do módulo Modelo 


\subsection{Módulo PAM}

Este módulo está baseado nos artefatos da Semiótica Organizacional (SO). Seu objetivo é apoiar a análise e clarificação de problemas considerando desde aspectos sociais até os elementos técnicos que o envolvem. Está composto por quatro componentes, que estão orientados ao trabalho colaborativo (ver Figura 6). Cada uma das abas dá acesso a um dos artefatos descritos a seguir:

Problema proposto: este artefato permite ao professor cadastrar o problema a ser resolvido, identificando o núcleo temático do qual faz parte e uma breve descrição do problema.

Partes Interessadas: com este artefato da SO é possível identificar os elementos que têm alguma relação direta ou indireta com o problema. Todas as partes interessadas inseridas por qualquer integrante do grupo, são refletidas e visualizadas por todos os membros do grupo. A ferramenta permite registrar a descrição da parte interessada e o usuário que a cadastrou. Algumas funcionalidades adicionais que podem ser usadas durante a análise do problema incluem a categorização das partes interessadas e a possibilidade de mudar uma parte interessada entre camadas (operação, contribuição, fonte, mercado e comunidade), conforme ilustra a Figura 4.

Quadro de Avaliação: este artefato da SO permite antecipar possíveis problemas às idéias iniciais de solução e soluções alternativas, agrupadas pelas camadas de partes interessadas (operação, contribuição, fonte, mercado e comunidade). O módulo permite ainda escolher um problema ou uma solução e deslocá-lo entre camadas ou entre problemas e soluções. A Figura 6 ilustra esse artefato.

Framework Semiótico: artefato da SO que permite identificar os requisitos do problema em seis camadas (mundo social, pragmática, semântica, sintática, empírica e física), conforme ilustrado na Figura 7. Os elementos identificados podem ser inseridos como requisito ou pergunta com uma descrição complementar. Este componente possibilita interação entre requisitos e mudá-los de camadas.

\subsection{Módulo Documentos}

No modelo ACBP é fundamental o caminho percorrido na resolução do problema e as diversas atividades realizadas pelos estudantes para desenvolver a solução. Neste módulo foram criados três componentes, que permitem a documentação de atividades relevantes durante o processo (ver Figura 7). Os componentes desse módulo estão representados nas abas da interface de usuário:

Atas: depois de cada reunião de grupo é importante cadastrar a síntese daquela atividade. Isto permite que os estudantes possam ter disponível um resumo de tudo o que foi discutido e decidido. Uma ata estará disponível para o seu preenchimento só depois que uma reunião programada esteja encerrada. O grupo poderá cadastrar: um resumo da reunião, uma descrição mais completa sobre os acontecimentos ou temas abordados na reunião, conclusões da reunião e novas tarefas atribuídas aos membros.

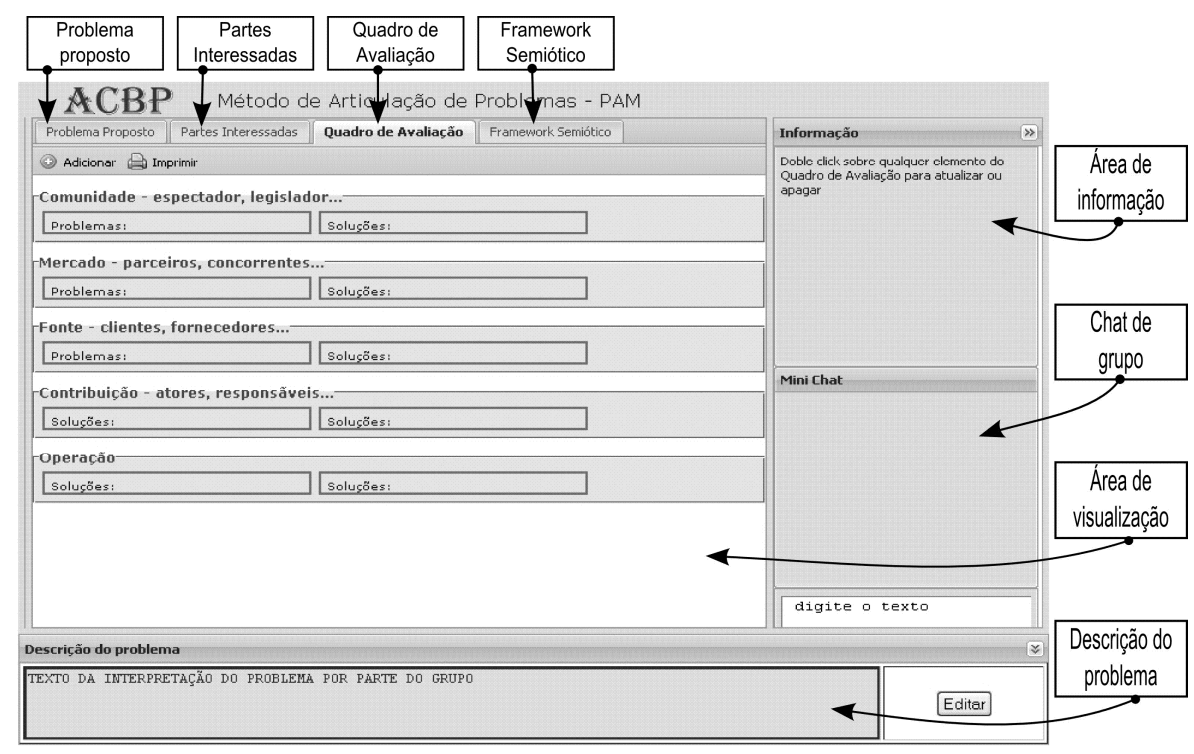

Figura 6: Interface de usuário da Ferramenta Quadro de Avaliação do módulo PAM 


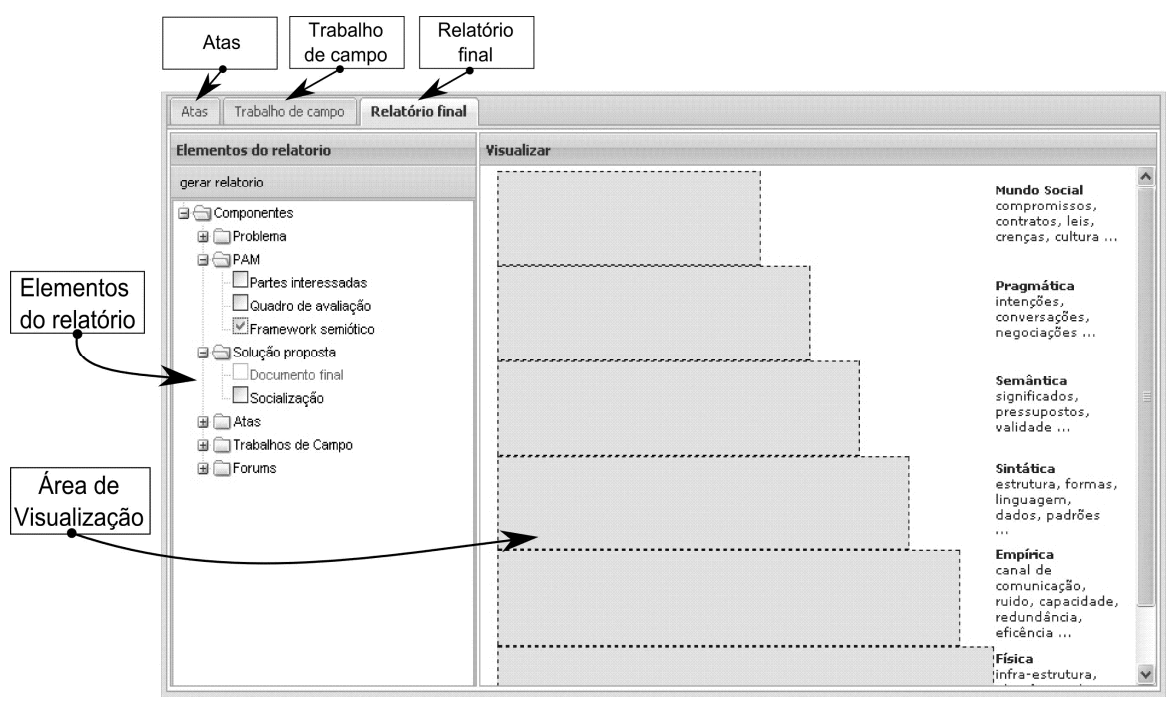

Figura 7: Interface de usuário da ferramenta Relatório Final do módulo Documentos

Trabalho de campo: todas as atividades desenvolvidas individualmente pelos integrantes podem ser registradas neste componente. Isto permitirá ter disponíveis informações sobre o que cada membro está fazendo, os aportes do grupo e permitirá ao professor identificar as contribuições e a participação de cada integrante no processo.

Relatório final: considera-se que parte importante do modelo ACBP é o processo que leva à solução do problema. Este componente possibilita a criação de um relatório final com os elementos criados pelo grupo durante o processo. Pretende-se também que tal relatório seja publicado e disponibilizado para futuras consultas, possibilitando a criação de um banco de problemas resolvidos.

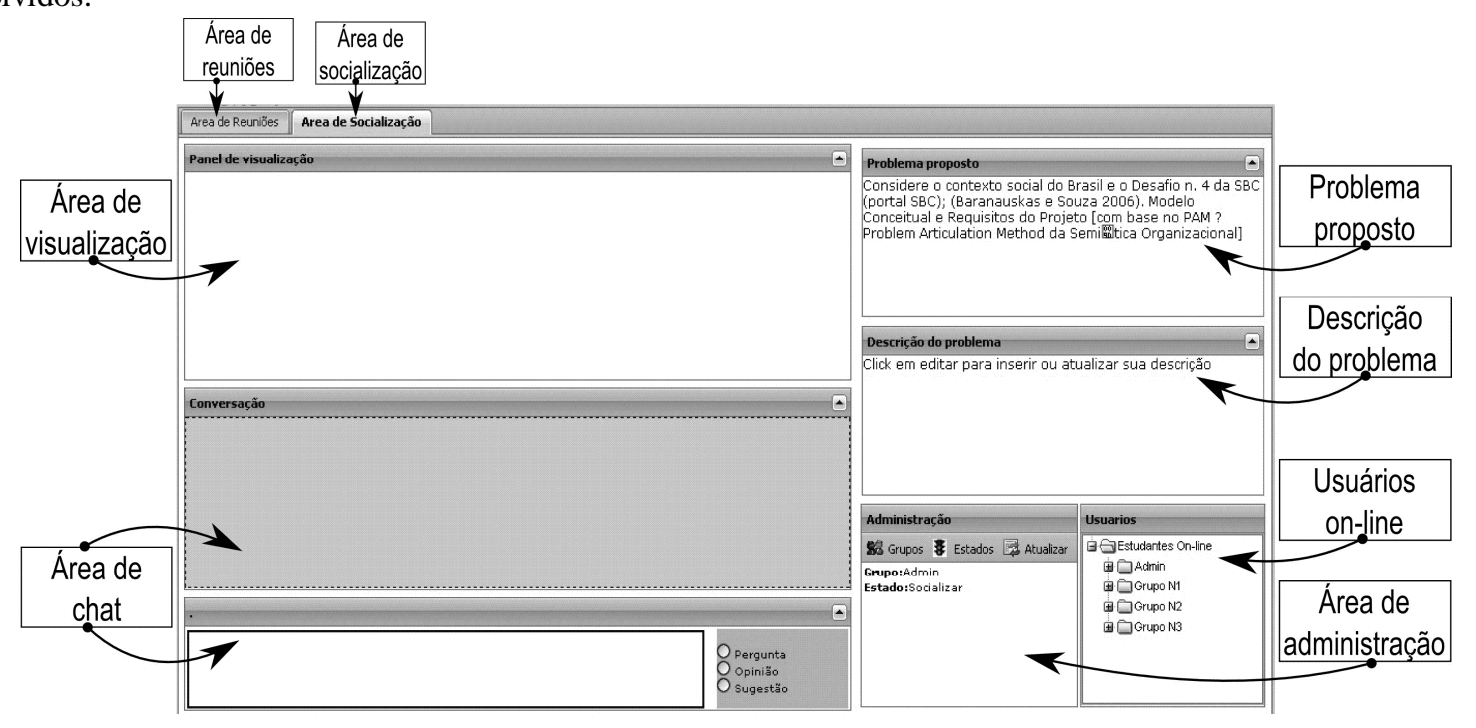

Figura 8: Interface de usuário da ferramenta Área de Socialização do módulo Reuniões

\subsection{Módulo Reuniões}

Considerando que o chat fornecido pelo Sakai só funciona ao nível de turma e não permite personalização, identificou-se a necessidade de se criar dois novos componentes como esquemas de comunicação personalizados de acordo com o modelo ACBP, para articular algumas características que permitissem restringir e canalizar cada participação (ver Figura 8).

A Figura 8 apresenta a funcionalidade do módulo Reuniões distribuído em duas abas: 
Área de Reuniões: este componente permite programar reuniões de grupo. Qualquer membro do grupo poderá convocar uma reunião. Para tal ele deverá preencher os campos da data da reunião, o tema central da reunião e listar um conjunto de atividades ou subtópicos que serão discutidos.

Todos podem participar na conversação postando conteúdos de texto, mas cada membro deverá classificar cada aporte em qualquer uma das cinco categorias disponíveis (expressão livre ou sem classificação, opinião, contribuição, desacordo e novo tema). Isto possibilita classificar o diálogo em um conjunto de idéias que possam ser mais produtivas segundo o modelo de fóruns estruturados [3, 19, 25].

Área de socialização: através deste componente, cada grupo poderá apresentar seu trabalho. O professor é a pessoa encarregada de coordenar esta atividade; ele poderá escolher o grupo que apresenta, o tempo para cada uma das fases (apresentação, sugestões, perguntas e respostas) e também poderá fazer parte do diálogo.

A ferramenta está composta por uma área de visualização onde está disponível o artefato apresentado, uma área de chat para comunicação com a possibilidade de classificar os textos postados (opinião, sugestão, pergunta). Os usuários que estão apresentando têm uma área adicional para administrar os artefatos a serem visualizados por todos os demais.

\subsection{Outras ferramentas}

Além dos componentes mencionados, também foram desenvolvidos componentes adicionais que fazem parte das funcionalidades do modelo e estão disponíveis nos módulos PAM e Documentos. São eles:

Informação: este componente permite apresentar informações sobre o uso das ferramentas ou detalhes dos artefatos e seus elementos.

Editor colaborativo para descrição do problema: este componente permite que os membros de cada grupo possam criar colaborativamente uma descrição própria sobre a sua interpretação do problema, que permitirá nortear o processo.

Chat de grupo: permite uma comunicação rápida e direta entre os estudantes que fazem parte do grupo de trabalho, mantendo sempre um histórico dos textos postados durante todo o dia.

\section{Uso preliminar do ACBP-Sakai}

O ambiente ACBP-Sakai foi testado em diferentes momentos de seu processo de desenvolvimento. Inicial- mente as ferramentas desenvolvidas no módulo PAM foram utilizadas no contexto da oficina número 1 do projeto e-Cidadania ${ }^{8}$, para testar o comportamento das ferramentas em uma situação real de discussão e clarificação de problema.

As ferramentas foram utilizadas de forma colaborativa pelos pesquisadores da equipe do e-Cidadania. Como resultado desse uso preliminar foram identificados alguns ajustes necessários principalmente pelo alto volume de informação coletada. Para tratar os aspectos apontados no caso real de uso, foi realizado um conjunto de melhorias relacionadas à flexibilidade da interação com os artefatos do PAM. De forma geral, o Módulo PAM e suas ferramentas atenderam as necessidades do processo de clarificação de problema e sua documentação na oficina do referido Projeto, de forma bastante efetiva. A Figura 9 ilustra o artefato Partes Interessadas resultado desse uso.

O ambiente ACBP-Sakai como um todo foi utilizado em duas disciplinas do Instituto de Computação (IC) UNICAMP: MO825(B) ${ }^{9}$ ministrada a estudantes de pósgraduação e $\mathrm{MC} 750(\mathrm{~F})^{10} / \mathrm{MO} 645(\mathrm{~B})^{11}$ integradas como uma disciplina e ministrada a estudantes de graduação e pós-graduação em conjunto. A dinâmica das disciplinas foi proposta de forma a articular teoria e pratica em atividades de grupo, permitindo o desenvolvimento progressivo de conteúdos. As atividades de cada disciplina estiveram baseadas na resolução de problemas usando o modelo ACBP e o ambiente ACBP-Sakai. Discussão detalhada dos resultados do uso do ambiente, seu modelo e ferramentas nessas disciplinas está apresentada em Melo-Solarte (2009) [17].

Embora as disciplinas tenham sido ministradas presencialmente, parte das atividades foi realizada a distância usando as ferramentas fornecidas pelo ambiente, permitindo avaliar o funcionamento de cada ferramenta em um contexto real. Isto, por sua vez, permite vislumbrar o ACBP como um modelo que pode ser considerado no desenvolvimento de diferentes cursos, presenciais ou a distância.

Atualmente ACBP-Sakai continua em uso como ambiente de apoio em disciplinas de graduação e pósgraduação. Além disso, está sendo previsto seu uso em formação a distância de professores no projeto PROESP/CAPES ${ }^{12}$, em temáticas relacionadas à Educação Inclusiva.

\footnotetext{
${ }^{8}$ http://styx.nied.unicamp.br:8080/ecidadania

9 Tópicos em Interfaces de Usuário: Semiótica da Interação HumanoArtefato Digital.

${ }^{10}$ Construção de Interfaces Homem-Computador.

${ }^{11}$ Projeto de Interfaces de Usuário.

${ }^{12} \mathrm{http} / / /$ styx.nied.unicamp.br:8080/todosnos/
} 


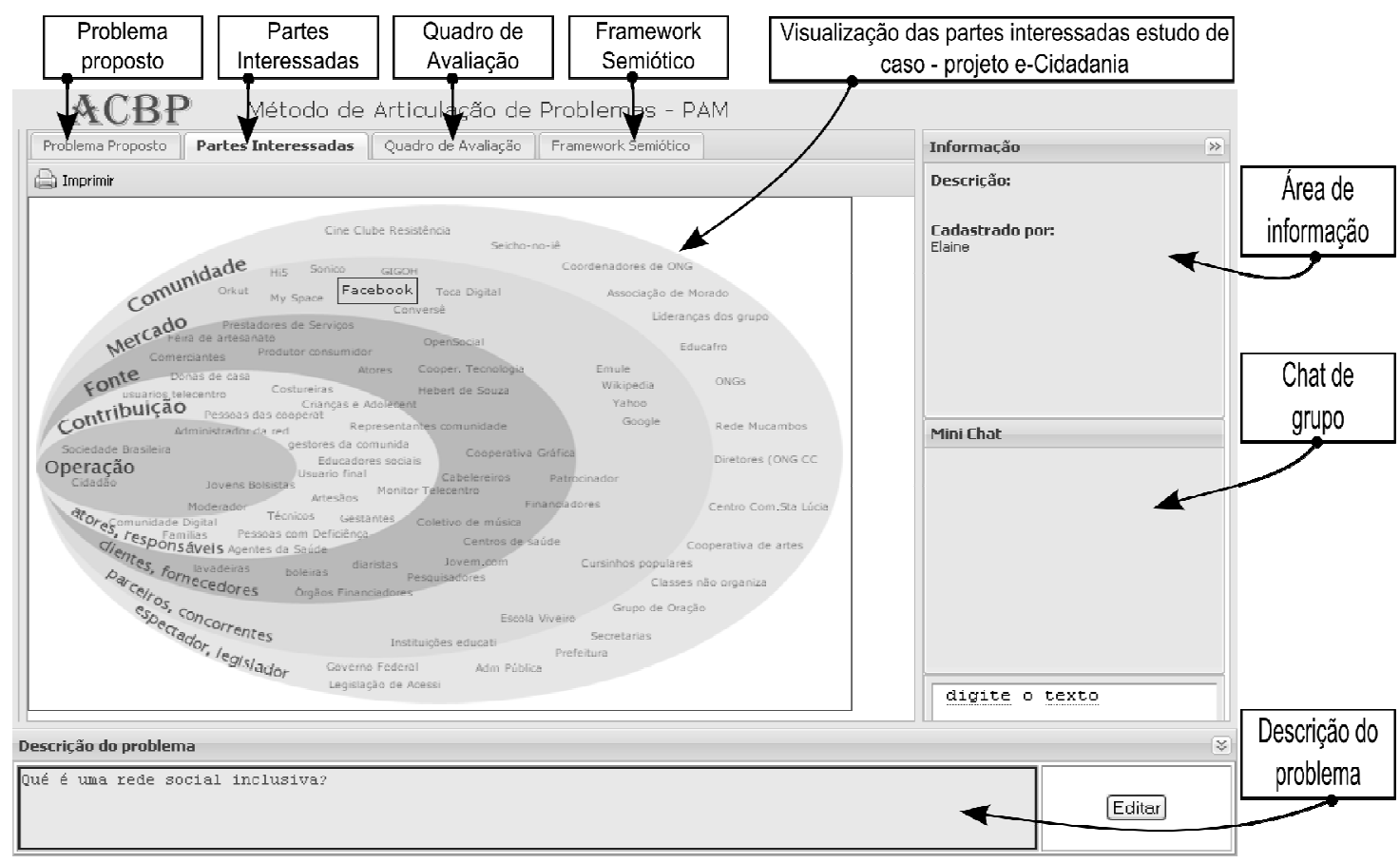

Figura 9: Interface de usuário da ferramenta Partes Interessadas do projeto e-Cidadania

\section{Conclusão}

Quando o problema sobre o qual os estudantes estão trabalhando é complexo, eles podem se beneficiar de colaboração em grupo para entendê-lo sob várias perspectivas, gerar e testar idéias de soluções e discutir seus impactos potenciais. Por outro lado, literatura tem mostrado que quando o aprendizado colaborativo é efetivo os estudantes podem resolver problemas muito mais complexos e chegar a um entendimento mais sofisticado dos domínios envolvidos. Este artigo contribui com a apresentação e discussão desses aspectos na perspectiva do modelo ACBP.

O trabalho envolveu a elaboração sobre PBL e CSCL de uma proposta conceitual para uma abordagem ao ensino/aprendizagem que é baseada no compartilhamento de significados e discussão coletiva sobre o problema e suas soluções. A partir de análise de vários LMS esse modelo conceitual foi desenvolvido na forma de ferramentas computacionais e integrado ao ambiente de ensino a distância Sakai. Um novo ambiente para ensino/aprendizado baseado em problemas e mediado por tecnologia de informação e comunicação, ACBP-Sakai foi criado integrando novos módulos e ferramentas necessárias aos requisitos do modelo conceitual. Resultados preliminares de uso em contexto real de clarificação e discussão de problema mostrou a efetividade das ferra- mentas. O ambiente completo está atualmente em uso em disciplinas de curso superior.

\section{Referências}

[1] ITESM, Aprendizaje basado en problemas como técnica didáctica, dirección de investigación y desarrollo educativo vicerrectoría académica, Instituto Tecnológico y de Estudios Superiores de Monterrey, 2002. Disponível em: <http://www.ub.es/mercanti/abp.pdf >. Acesso em: Fevereiro de 2008

[2] H. Barrows, A Taxonomy of Problem-Based Learning Methods. Medical Education, vol. 20, páginas 481-486, 1986

[3] A. Calvani, A. Fini, M. C. Pettenati, L. Sarti, M. Masseti, Design of Collaborative Learning Environments: bridging the gap between CSCL theories and Open Source Platforms, Journal of e-Learning and Knowledge Society, 2006.

[4] T. Cameron, H. S. Barrows, S. M. Crooks, Distributed problem-based learning at Southern Illinois University School of Medicine. CSCL '99: Proceedings of the 1999 conference on Computer support for collaborative learning, 
International Society of the Learning Sciences, Palo Alto - California, páginas 10, 1999.

[5] P. M. Carvalho, Modelo de Uso da Tecnologia de Informação no Suporte ao Processo de Ensino-Aprendizagem Baseado em Problemas no Curso Médico: Desenvolvimento e Avaliação. Tese de Doutorado, Universidade Estadual de Campinas. Faculdade de Engenharia Elétrica e de Computação, 2002.

[6] D. L. Cogburn, L. Zhang, M. Khothule, Going global, locally: the socio-technical influences on performance in distributed collaborative learning teams, SAICSIT '02: Proceedings of the 2002 annual research conference of the South African institute of computer scientists and information technologists on Enablement through technology, South African Institute for Computer Scientists and Information Technologists, Republic of South Africa, páginas 52-64, 2002.

[7] A. Dimitracopoulou, Designing collaborative learning systems: current trends \& future research agenda, CSCL '05: Proceedings of th 2005 conference on Computer support for collaborative learning, International Society of the Learning Sciences, páginas 115-124, 2005.

[8] L. A. G. González, Um modelo conceitual para aprendizagem colaborativa baseada na execução de projetos pela web, Tese de Doutorado, Universidade de São Paulo - Escola Politécnica (EP), 2005.

[9] B. R. Gómez, Aprendizaje basado en problemas (ABP): una innovación didáctica para la enseñanza universitaria. Educación y educadores. vol. 8, páginas 9-20, 2005.

[10] W. Hung, J. Lockard, Students' perception of knowledge activation on a guided collaborative problem solving organizer, ICLS '06: Proceedings of the 7th international conference on Learning sciences, International Society of the Learning Sciences, Páginas 270-276, 2006.

[11] T. Greening, J. Kay, J. H. Kingston, K. Crawford, Results of a PBL trial in first-year computer science. ACSE '97: Proceedings of the 2nd Australasian conference on computer science education, New York, Páginas 201-206, 1996.

[12] J. Iglesias, El aprendizaje basado en problemas en la formación inicial de docentes Perspectivas, 32 (3), páginas 319-332, 2002.
[13] C. Jones, L. Dirckinck-Holmfeld, B. Lindtröm, CSCL - the next ten years: a view from Europe, CSCL '05: Proceedings of th 2005 conference on Computer support for collaborative learning, International Society of the Learning Sciences, páginas 237-246, 2005.

[14] J. L. Kolodner, K; Nagel, The design discussion area: a collaborative learning tool in support of learning from problem-solving and design activities, CSCL '99: Proceedings of the 1999 conference on Computer support for collaborative learning, International Society of the Learning Sciences, páginas 37, 1999.

[15] K. Liu, Semiotics in Information Systems Engineering, MIT Cambridge University Press, New York - USA, 2000.

[16] D. S. Melo-Solarte, M. C. C. BARANAUSKAS, Uma Abordagem para EaD Baseada em Resolução de Problemas. In: SBIE 2008 Simpósio Brasileiro de Informática na Educação, 2008, Fortaleza. Anais do SBIE 2008 XIX Simpósio Brasileiro de Educação, 2008. p. 716-725.

[17] D. S. Melo-Solarte, Aprendizagem Colaborativa Baseada em Problemas - ACBP: Modelo Conceitual e Ferramentas, Dissertação de Mestrado, Universidade Estadual de Campinas - Instituto de Computação (IC-UNICAMP), Abril 2009.

[18] P. Morales, V. Landa, Aprendizaje Basado em Problemas Problem - Based Learning. Theoria, Vol 13, páginas 145-157, 2004.

[19] M. Pimentel, H. Fuks,C. J. P. de Lucena, Mediated chat development process: avoiding chat confusion on educational debates, CSCL '05: Proceedings of th 2005 conference on Computer support for collaborative learning, International Society of the Learning Sciences, 499-503, 2005.

[20] G. Stahl, Group cognition: the collaborative locus of agency in CSCL, CSCL '05: Proceedings of th 2005 conference on Computer support for collaborative learning, International Society of the Learning Sciences, Taipei - Taiwan, páginas 632-640, 2005.

[21] G. Stahl, T. Koschmann, D. Suthers, Computersupported collaborative learning: An historical perspective, Cambridge handbook of the learning sciences, Cambridge University Press, páginas 409-426, 2006. 
[22] R. Stamper, "Social Norms in requirements analysis an outline of MEASUR". In Requirements Engineering, Technical and Social Aspects, Academic Press, 1993.

[23] H. Tsuji, R. H. Aguilar-da-Silva, Experience with a problem-based curriculum implemented in the endocrine system unit in the 2nd grade medical course at Marília Medical School Famema Arquivos Brasileiros de Endocrinologia \& Metabologia, 48(4), páginas 535 -543, 2004.

[24] A. Weinberger, D. Clark, G. Erkens, V. Sampson, K. Stegmann, J. Janssen, J. Jaspers, G. Kanselaar, F. Fischer, rgumentative knowledge construction in CSCL, ICLS '06: Proceedings of the 7th international conference on Learning sciences, International Society of the Learning Sciences, páginas 1094-1100, 2006.

[25] J. Yeo, S. C. Tan, Y. Lee. A learning journey in problem-based learning. ICLS '06: Proceedings of the 7th international conference on Learning sciences, International Society of the Learning Sciences, páginas 859-865, 2006. 